\title{
$\mathrm{Ag}$ 与 $\mathrm{Ag}_{2} \mathrm{O}$ 协同增强 $\mathrm{TiO}_{2}$ 光催化制氢性能的研究
}

\author{
王 苹，李心宇，时占领，李海涛 \\ (武汉理工大学 化学化工与生命科学学院, 武汉 430070)
}

摘 要: 本研究采用两步法制备了电子助剂 $\mathrm{Ag}$ 和界面活性位点 $\mathrm{Ag}_{2} \mathrm{O}$ 共修饰的高效 $\mathrm{TiO}_{2}$ 光催化剂 $\left(\mathrm{TiO}_{2} / \mathrm{Ag}-\mathrm{Ag}_{2} \mathrm{O}\right)$ : 首先用光沉积法将 $\mathrm{Ag}$ 负载在 $\mathrm{TiO}_{2}$ 表面 $\left(\mathrm{TiO}_{2} / \mathrm{Ag}\right.$ ), 再经过低温䍩烧法使部分 $\mathrm{Ag}$ 原位生成 $\mathrm{Ag}_{2} \mathrm{O}$ 。紫外光照射 $\mathrm{TiO}_{2}$ 时, 激发产生的电子被助剂 $\mathrm{Ag}$ 捕获后快速传输到 $\mathrm{Ag}_{2} \mathrm{O}$ 上, 电子把 $\mathrm{Ag}_{2} \mathrm{O}$ 界面产氢活性位点从溶液中所捕获的氢离 子还原成氢气, $\mathrm{Ag}$ 和 $\mathrm{Ag}_{2} \mathrm{O}$ 的协同作用加快了 $\mathrm{TiO}_{2}$ 上光生电子的转移和界面产氢反应, 从而提高了 $\mathrm{TiO}_{2} / \mathrm{Ag}-\mathrm{Ag}_{2} \mathrm{O}$ 制氢性能。在 $300{ }^{\circ} \mathrm{C}$ 㷽烧温度下制备的 $\mathrm{TiO}_{2} / \mathrm{Ag}-\mathrm{Ag}_{2} \mathrm{O}$ 光催化剂制氢速率最高, 达到 $75.20 \mu \mathrm{mol} / \mathrm{h}$, 分别是 $\mathrm{TiO}_{2}(3.59 \mu \mathrm{mol} / \mathrm{h})$ 和 $\mathrm{TiO}_{2} / \mathrm{Ag}(41.13 \mu \mathrm{mol} / \mathrm{h})$ 的 21.0 倍和 1.8 倍。本研究为光催化制氢材料的设计和制备提供了有益 的参考。

关 键 词: $\mathrm{TiO}_{2}$; 电子助剂; 界面活性位点; 协同作用; 光催化制氢

中图分类号: O643 文献标识码: A

\section{Synergistic Effect of $\mathrm{Ag}$ and $\mathrm{Ag}_{2} \mathrm{O}$ on Photocatalytic $\mathrm{H}_{2}$-evolution Performance of $\mathrm{TiO}_{2}$}

\author{
WANG Ping, LI Xinyu, SHI Zhanling, LI Haitao
}

(School of Chemistry, Chemical Engineering and Life Science, Wuhan University of Technology, Wuhan 430070, China)

\begin{abstract}
Highly efficient $\mathrm{TiO}_{2}$ photocatalysts $\left(\mathrm{TiO}_{2} / \mathrm{Ag}-\mathrm{Ag}_{2} \mathrm{O}\right)$ co-modified by $\mathrm{Ag}$ as electron cocatalysts and $\mathrm{Ag}_{2} \mathrm{O}$ as interfacial catalytic active sites were synthesized via a two-step process including the initial photoinduced deposition of metallic Ag nanoparticles on the $\mathrm{TiO}_{2}$ surface $\left(\mathrm{TiO}_{2} / \mathrm{Ag}\right)$ and the following in situ oxidation of partial $\mathrm{Ag}$ into $\mathrm{Ag}_{2} \mathrm{O}$ by low-temperature calcination. Ag nanoparticles function as effective electron cocatalysts for the steady capture and rapid transportation of photogenerated electrons from $\mathrm{TiO}_{2}$ surface to $\mathrm{Ag}_{2} \mathrm{O}$, while the adsorbed $\mathrm{H}^{+}$ions from solution to $\mathrm{Ag}_{2} \mathrm{O}$ as the interfacial catalytic active sites are reduced into $\mathrm{H}_{2}$. The synergistic effect of $\mathrm{Ag}$ and $\mathrm{Ag}_{2} \mathrm{O}$ can accelerate the electrons transfer and promote the rapid $\mathrm{H}_{2}$-evolution reaction for enhanced photocatalytic $\mathrm{H}_{2}$-evolution performance of $\mathrm{TiO}_{2} / \mathrm{Ag}-\mathrm{Ag}_{2} \mathrm{O}$. The highest $\mathrm{H}_{2}$-evolution rate of the resultant $\mathrm{TiO}_{2} / \mathrm{Ag}-\mathrm{Ag}_{2} \mathrm{O}$ calcinated at $300{ }^{\circ} \mathrm{C}$ reached $75.20 \mu \mathrm{mol} / \mathrm{h}$, which was higher than those of the $\mathrm{TiO}_{2}(3.59 \mu \mathrm{mol} / \mathrm{h})$ and $\mathrm{TiO}_{2} / \mathrm{Ag}$ $(41.13 \mu \mathrm{mol} / \mathrm{h})$ by 21.0 and 1.8 times, respectively. This study provides a new strategy for the design and synthesis of highly efficient photocatalytic $\mathrm{H}_{2}$-evolution materials.
\end{abstract}

Key words: $\mathrm{TiO}_{2}$; electron cocatalyst; interfacial catalytic active site; synergistic effect; photocatalytic $\mathrm{H}_{2}$ evolution

收稿日期: 2019-09-04; 收到修改稿日期: 2019-12-04

基金项目：国家自然科学基金(51872221); 国家级大学生创新创业训练计划项目(20181049715025)

National Natural Science Foundation of China (51872221); National Undergraduate Innovation And Entrepreneurship Training Program (20181049715025)

作者简介: 王 苹(1970-), 女, 教授. E-mail: wangping0904@whut.edu.cn WANG Ping (1970-), female, professor. E-mail: wangping0904@whut.edu.cn 
随着社会生产力的发展, 人类对能源的需求量 日益增大，但是能源与环境平衡的问题也不容忽 视。在能源危机和环境污染的双重压力下, 各国科 学家纷纷将研究的重点转向新型清洁能源的开 发 ${ }^{[1-4]}$ 。氢能因产热高、储量大、清洁无污染等优点 受到了各国科学家的青睐 ${ }^{[5-6]}$ 。在各类制氢技术中, 由 Fujishima 等 ${ }^{[7-10]}$ 开创的光催化制氢技术具有反应 条件温和、能耗低、操作简便等优势, 成为一项具 有巨大应用潜力的新型制氢技术。作为光催化剂, $\mathrm{TiO}_{2}$ 的性能稳定、无毒, 并具有抗光腐蚀等优点, 但光生电子-空穴对极易复合, 导致其制氢效率较 低 ${ }^{[11-12]}$ 。目前可以通过形貌控制 ${ }^{[13]}$ 、离子掺杂 ${ }^{[14]}$ 、 半导体复合 ${ }^{[15]}$ 和助剂修饰 ${ }^{[16]}$ 等方法对 $\mathrm{TiO}_{2}$ 改性。 其中, 助剂表面修饰用量少、条件温和, 并且对主 体材料结构影响很小, 是一种常见、有效的改性 手段。

已有研究 ${ }^{[17-19]}$ 表明, 以金属纳米粒子作为电子 助剂修饰改性 $\mathrm{TiO}_{2}$ 是一种提高光催化性能的有效 方法。但在大多数情况下, 金属材料(除 Pt 以外)本 身都不能作为有效的界面催化活性位点, 导致迁移 到金属表面的电子不能及时与吸附在材料表面的 $\mathrm{H}^{+}$发生还原反应生成 $\mathrm{H}_{2}$, 使金属修饰的 $\mathrm{TiO}_{2}$ 光催 化材料表现出较低的界面析氢速率, 并成为整个反 应过程中的控速步骤，最终制约了整体制氢效率。 最近研究表明 ${ }^{[20-21]}$, 在金属电子助剂表面增加金属 氧化物或硫化物等作为界面催化活性位点协同修饰 改性光催化剂是一种提高光催化性能的有效方法。 但由于一般在半导体光催化材料表面修饰的金属颗 粒比较小, 往往是纳米级别的小颗粒, 很难大量吸 附作为界面活性位点的基团或离子。而且，如果金 属电子助剂和界面活性位点之间是以物理吸附方式 结合, 相互作用力较弱, 结合不够牢固, 仍然会影 响光生电子的快速传输以及随后的界面催化产氢反 应, 不能明显改善光催化制氢性能。因此, 发展新型 界面催化活性位点, 促进其界面产氢催化反应, 对 于提高半导体/电子助剂光催化材料体系的制氢性 能至关重要。

在金属表面定向生成可作为界面催化活性位点 的物质, 可以使两者紧密结合, 进而改善电子的传 输。考虑到 $\mathrm{Ag}_{2} \mathrm{O}$ 有一定的吸氢能力, 而且可以在 $\mathrm{Ag}$ 与氧气发生反应时原位生成, 使 $\mathrm{Ag}_{2} \mathrm{O}$ 与 $\mathrm{Ag}$ 之 间存在很强的亲和力, 本研究设计了在 $\mathrm{TiO}_{2}$ 光催化 材料表面光沉积 $\mathrm{Ag}$ 后煆烧再生成 $\mathrm{Ag}_{2} \mathrm{O}$ 的方法, 制 备了电子助剂 $\mathrm{Ag}$ 和界面活性位点 $\mathrm{Ag}_{2} \mathrm{O}$ 共修饰的 $\mathrm{TiO}_{2}$ 光催化材料 $\left(\mathrm{TiO}_{2} / \mathrm{Ag}-\mathrm{Ag}_{2} \mathrm{O}\right)$, 测试了 $\mathrm{TiO}_{2} / \mathrm{Ag}-$
$\mathrm{Ag}_{2} \mathrm{O}$ 光催化剂的制氢性能, 并研究了其光催化制 氢机理。

\section{1 实验方法}

\section{1 主要试剂}

商业 $\mathrm{P} 25 \mathrm{TiO}_{2}$ 购于 Degussa 公司, $\mathrm{AgNO}_{3}$ 、甲醇 和乙醇购于上海化学试剂公司, 均为分析纯。实验 过程中用水均为去离子水。

\section{2 样品制备}

\subsection{1 $\mathrm{TiO}_{2}-\mathrm{C}$ 的制备}

将装有一定量的 $\mathrm{P} 25 \mathrm{TiO}_{2}$ 的坩埚放入马弗炉中, 然后以 $5{ }^{\circ} \mathrm{C} / \mathrm{min}$ 的速率升到 $550{ }^{\circ} \mathrm{C}$ 并煅烧 $2 \mathrm{~h}$, 冷却 后取出坩埚,得到 $\mathrm{TiO}_{2}-\mathrm{C}$ 。

\subsection{2 $\mathrm{TiO}_{2} / \mathrm{Ag}$ 的制备}

在三颈烧瓶中将 $200 \mathrm{mg} \mathrm{TiO}_{2}$ 粉末分散到 $80 \mathrm{~mL} \mathrm{10vol \%}$ 的甲醇溶液中, 磁力搅拌 $10 \mathrm{~min}$, 得 到均匀分散的白色悬浊液; 随后, 在上述 $\mathrm{TiO}_{2}$ 悬浊 液中缓慢滴加 $186 \mu \mathrm{L} 0.10 \mathrm{~mol} / \mathrm{L}$ 的 $\mathrm{AgNO}_{3}$ 溶液。摚 拌混合液 $60 \mathrm{~min}$, 通氮气 $15 \mathrm{~min}$, 然后用 4 个紫外 光灯 (3 W, $365 \mathrm{~nm}$ ) 照射上述悬浊液, 触发 $\mathrm{Ag}^{+}$的还 原反应。照射 $60 \mathrm{~min}$ 后, 经过滤、洗涤和干燥, 得 到棕红色的 $\mathrm{TiO}_{2} / \mathrm{Ag}$ 复合光催化剂。

\subsection{3 $\mathrm{TiO}_{2} / \mathrm{Ag}-\mathrm{Ag}_{2} \mathrm{O}$ 的制备}

将 $80 \mathrm{mg} \mathrm{TiO}_{2} / \mathrm{Ag}$ 样品放置于带有盖子的瓷舟 中, 放入马弗炉中炦烧，以 $10{ }^{\circ} \mathrm{C} / \mathrm{min}$ 的速度上升至 设定温度 $\left(200,250,300,350,400{ }^{\circ} \mathrm{C}\right)$ 。制烧结束后, 立即从炉子中取出产物并密封, 将其冷却至室温, 即得 $\mathrm{TiO}_{2} / \mathrm{Ag}-\mathrm{Ag}_{2} \mathrm{O}$ 光催化复合材料, 标记为 $\mathrm{TiO}_{2} / \mathrm{Ag}-\mathrm{Ag}_{2} \mathrm{O}(x)$, 其中 $x=200,250,300,350,400$, 表示煅烧温度。

\section{3 光催化制氢性能测试}

参考本课题组的方法 ${ }^{[16]}$ 对材料进行制氢性能 测试, 具体操作步骤为: 在 $100 \mathrm{~mL}$ 的三口平底烧瓶 中, 将 $50 \mathrm{mg}$ 光催化剂样品分散到 $80 \mathrm{~mL} 25 \mathrm{vol} \%$ 的 乙醇溶液牺牲剂中, 通氮气 $15 \mathrm{~min}$ 。将密封的反应 容器放置在距离光源 $(4$ 个 $365 \mathrm{~nm}$ 的 LED 灯) $1 \mathrm{~cm}$ 的位置进行照射，同时进行摚拌。每间隔 $30 \mathrm{~min}$ 测 1 次氢气含量, 测试 $120 \mathrm{~min}$, 直至产氢稳定。气相 色谱仪 (岛津 $\mathrm{GC}-2014 \mathrm{C}$ ) 的载气为氮气, 配置 TCD 检测器和 $0.5 \mathrm{~nm}$ 分子篎毛细管柱。

\section{4 电化学测试}

采用标准三电极系统电化学工作站(CHI660E) 采集电化学数据, 对电极为 $\mathrm{Pt}$, 参比电极为 $\mathrm{CHI} 150$ 型 $\mathrm{Ag} / \mathrm{AgCl}$, 电解质溶液为 $0.5 \mathrm{~mol} / \mathrm{L} \mathrm{Na}_{2} \mathrm{SO}_{4}$ 溶液。 
采用旋涂方法将光催化剂涂布在导电玻璃(FTO)的 表面制备工作电极, 具体步骤为: 在由 $1.5 \mathrm{~mL}$ 的荎 酚 D-520 溶液 $(5 \mathrm{wt} \%$ )和 $1 \mathrm{~mL}$ 无水乙醇制得的混合 溶液中加入 $4 \mathrm{mg}$ 样品, 然后使用旋转涂膜仪将超声 后的悬浮液均匀涂抹在 FTO 上, 室温下放置 $1 \mathrm{~h}$ 后, 在 $40{ }^{\circ} \mathrm{C}$ 下烘 $24 \mathrm{~h}$ 。工作电极 $(J-V)$ 的线性扫描范围 为 $0 \sim-1.6 \mathrm{~V}$, 扫描速度为 $0.001 \mathrm{~V} / \mathrm{s}$ 。测试工作电极 的光电流 $(i-t)$ 光源为 $\operatorname{LED}$ 灯 $(3 \mathrm{~W}, \lambda=365 \mathrm{~nm}$, $80 \mathrm{~mW} / \mathrm{cm}^{2}$ ), 电压为 $0.5 \mathrm{~V}$, 循环光照 4 次, 每次光 照间隔 $60 \mathrm{~s}$ 。测试工作电极的电化学阻抗的初始电 压为开路电势, 扫描范围为 $0.001 \sim 10^{6} \mathrm{~Hz}$ 。

\section{5 样品表征}

采用 $X$ 射线衍射仪(XRD, Rigaku Ultima III)测 定样品的晶型; 采用场发射扫描电子显微镜 (FESEM, JEOL JSM-7500)和透射电子显微镜(TEM, JEOL JEM-2100F)对样品的形貌特征进行研究观察; 采用紫外-可见分光光度计(UV-Vis, Shimadzu UV2450)、以 $\mathrm{BaSO}_{4}$ 为标样测试样品的紫外-可见漫反 射光谱; 采用 $X$ 射线光电子能谱仪(XPS, KRATOA $\mathrm{XSAM} 800, \mathrm{Al} \mathrm{K} \alpha$ 靶)检测样品表面化学元素, 标准 碳元素峰 C 1s $284.6 \mathrm{eV}$ 为所有样品元素结合能的参 考标准。采用日本 Hitachi 公司的 F-7000 型荧光光 谱仪测定样品的苂光光谱，激发波长为 $350 \mathrm{~nm}$ 。

\section{2 结果与讨论}

\section{1 $\mathrm{TiO}_{2} / \mathrm{Ag}-\mathrm{Ag}_{2} \mathrm{O}$ 样品的合成}

图 1(A) 是通过光沉积法和煅烧法制备 $\mathrm{TiO}_{2} / \mathrm{Ag}-\mathrm{Ag}_{2} \mathrm{O}$ 样品的合成示意图, 图 1(B) 是对应的 样品照片。首先，传统的锐钛矿和金红石混合晶型 P25 $\mathrm{TiO}_{2}$ 经过高温炦烧得到样品 $\mathrm{TiO}_{2}-\mathrm{C}$, 将其分散 到 $10 \mathrm{vol} \%$ 甲醇溶液中, 得到乳白色的悬浊液。随后, 通过光沉积法使 $\mathrm{AgNO}_{3}$ 溶液中的 $\mathrm{Ag}^{+}$原位还原 成 $\mathrm{Ag}$ 纳米颗粒并沉积在 $\mathrm{TiO}_{2}$ 表面, 悬浊液的颜色 也从乳白色变为棕红色(图 1(B)中 b), 得到 $\mathrm{TiO}_{2} / \mathrm{Ag}$
光催化剂的悬浮液。最后, 将制备的 $\mathrm{TiO}_{2} / \mathrm{Ag}$ 样品 分别在马弗炉中不同温度下煅烧，得到 $\mathrm{TiO}_{2} / \mathrm{Ag}-\mathrm{Ag}_{2} \mathrm{O}(x)$ 光催化剂。将所制得的 $\mathrm{TiO}_{2} / \mathrm{Ag}-\mathrm{Ag}_{2} \mathrm{O}$ 样品分散于乙醇溶液 (25vol\%)中, 悬 浊液分别为浅红色 $\left(\mathrm{TiO}_{2} / \mathrm{Ag}-\mathrm{Ag}_{2} \mathrm{O}(200)\right.$, 图 1(B)中 c)、浅蓝色 $\left(\mathrm{TiO}_{2} / \mathrm{Ag}-\mathrm{Ag}_{2} \mathrm{O}(300)\right.$, 图 1(B)中 d) 和灰白 色 $\left(\mathrm{TiO}_{2} / \mathrm{Ag}-\mathrm{Ag}_{2} \mathrm{O}(400)\right.$, 图 1(B)中 e), 样品对应悬浮 液的颜色随着衤烧温度的升高逐渐变浅。这是因为 $\mathrm{Ag}$ 在低温煆烧 $\left(100 \sim 400{ }^{\circ} \mathrm{C}\right)$ 的环境下部分被 $\mathrm{O}_{2}$ 氧 化为 $\mathrm{Ag}_{2} \mathrm{O}^{[22]}$, 而当温度达到 $300{ }^{\circ} \mathrm{C}$ 以上后, 生成的 $\mathrm{Ag}_{2} \mathrm{O}$ 又会分解生成 $\mathrm{O}_{2}$ 和 $\mathrm{Ag}$, 导致样品的颜色出现 相应变化。

\section{2 样品的微结构分析}

为确定所制备样品的晶体结构, 测定了不同样 品的 XRD 图谱(图 2)。 $\mathrm{TiO}_{2}-\mathrm{C}$ 中同时存在金红石型 $\mathrm{TiO}_{2}$ (JCPDS-99-0090) 和锐钛矿型 $\mathrm{TiO}_{2}$ (JCPDS-990008)的衍射峰，与原料 $\mathrm{P} 25$ 晶型一致 ${ }^{[12]}$ 。 $\mathrm{TiO}_{2} / \mathrm{Ag}$ 和 $\mathrm{TiO}_{2} / \mathrm{Ag}-\mathrm{Ag}_{2} \mathrm{O}(x)$ 的 XRD 衍射峰与 $\mathrm{TiO}_{2}-\mathrm{C}$ 相比 没有明显变化, 说明负载 $\mathrm{Ag}$ 和 $\mathrm{Ag}_{2} \mathrm{O}$ 对 $\mathrm{TiO}_{2}-\mathrm{C}$ 的 晶型以及晶化程度没有影响。比较 $\mathrm{TiO}_{2}-\mathrm{C}$ 和 $\mathrm{TiO}_{2} / \mathrm{Ag}$ 的金属 $\mathrm{Ag}$ 慢扫图(图 2(B)) 可见, $\mathrm{TiO}_{2} / \mathrm{Ag}$ 样

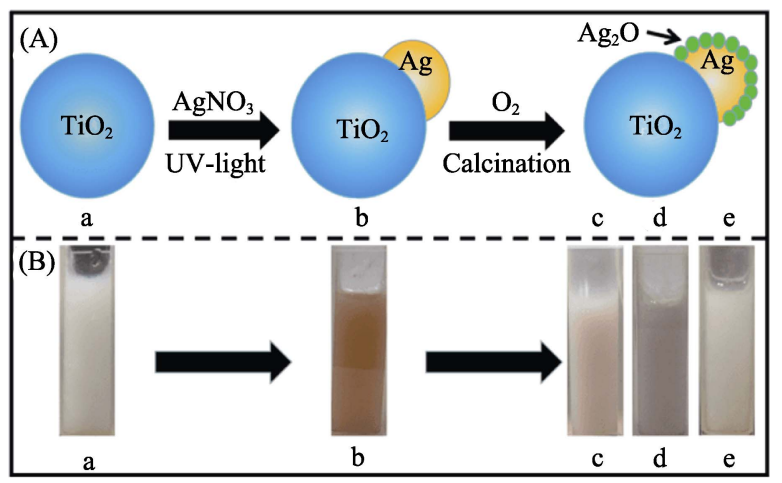

图 1 (A) $\mathrm{TiO}_{2} / \mathrm{Ag}-\mathrm{Ag}_{2} \mathrm{O}$ 合成示意图和(B)对应样品照片

Fig. 1 (A) Schematic diagram of preparation for $\mathrm{TiO}_{2} / \mathrm{Ag}-\mathrm{Ag}_{2} \mathrm{O}$ and (B) their corresponding photographs

(a) $\mathrm{TiO}_{2}-\mathrm{C}$; (b) $\mathrm{TiO}_{2} / \mathrm{Ag}$; (c) $\mathrm{TiO}_{2} / \mathrm{Ag}-\mathrm{Ag}_{2} \mathrm{O}\left(200\right.$ ); (d) $\mathrm{TiO}_{2} / \mathrm{Ag}-\mathrm{Ag}_{2} \mathrm{O}$ (300); (e) $\mathrm{TiO}_{2} / \mathrm{Ag}-\mathrm{Ag}_{2} \mathrm{O}(400)$
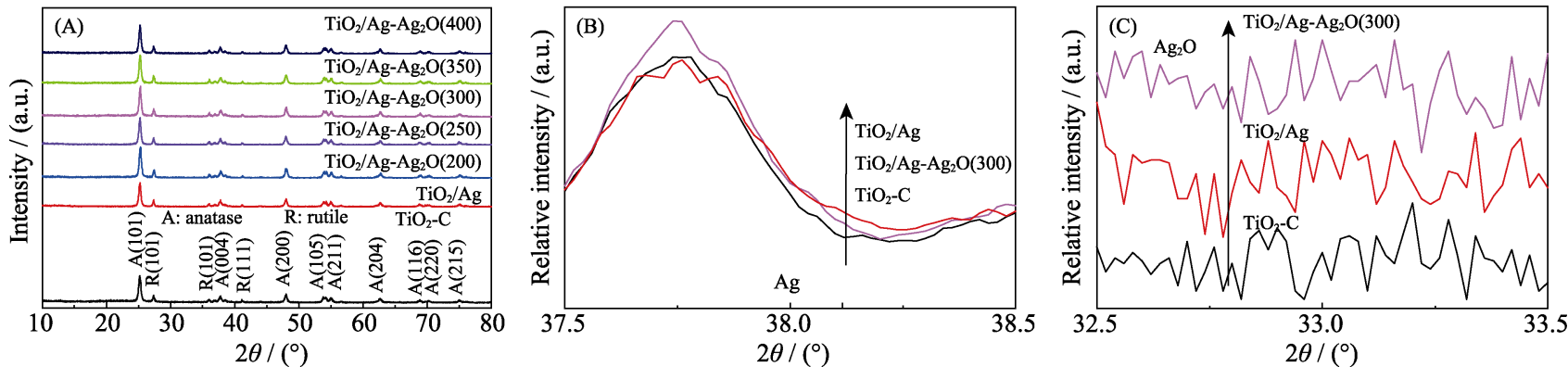

图 $2 \mathrm{TiO}_{2}-\mathrm{C} 、 \mathrm{TiO}_{2} / \mathrm{Ag}$ 和 $\mathrm{TiO}_{2} / \mathrm{Ag}-\mathrm{Ag}_{2} \mathrm{O}(x)$ 的(A) XRD 图谱、(B) 金属 $\mathrm{Ag}$ 的慢扫图谱和(C) $\mathrm{Ag}_{2} \mathrm{O}$ 的慢扫图谱

Fig. 2 (A) XRD patterns, (B) diffraction peaks of metallic $\mathrm{Ag}$ and (C) diffraction peaks of $\mathrm{Ag}_{2} \mathrm{O}$ for $\mathrm{TiO}_{2}-\mathrm{C}, \mathrm{TiO}_{2} / \mathrm{Ag}$ and $\mathrm{TiO}_{2} / \mathrm{Ag}-\mathrm{Ag}_{2} \mathrm{O}(x)$ 
品在金属 $\mathrm{Ag}$ 的特征衍射峰 $\left(2 \theta=38.1^{\circ}\right)$ 处有明显的增 强, $\mathrm{Ag}$ 助催化剂已经成功沉积在 $\mathrm{TiO}_{2}$ 表面 ${ }^{[19]}$ 。 $\mathrm{TiO}_{2} / \mathrm{Ag}-\mathrm{Ag}_{2} \mathrm{O}$ (300)在金属 $\mathrm{Ag}$ 的特征衍射峰处的强 度比 $\mathrm{TiO}_{2} / \mathrm{Ag}$ 有所减弱, 说明煅烧后样品中 $\mathrm{Ag}$ 含量 有所降低 ${ }^{[22]}$ 。进一步观察样品的 $\mathrm{Ag}_{2} \mathrm{O}$ 慢扫图 (图 2(C)) 发现, $\mathrm{TiO}_{2} / \mathrm{Ag}-\mathrm{Ag}_{2} \mathrm{O}(300)$ 样品中没有出现 明显的 $\mathrm{Ag}_{2} \mathrm{O}$ 衍射峰, 这可能是因为 $\mathrm{Ag}_{2} \mathrm{O}$ 的含量太 低而无法检出。

从场发射扫描电镜(图 3(A F)) 中发现, $\mathrm{TiO}_{2}-\mathrm{C}$ 、 $\mathrm{TiO}_{2} / \mathrm{Ag}$ 和 $\mathrm{TiO}_{2} / \mathrm{Ag}-\mathrm{Ag}_{2} \mathrm{O}(x)$ 的颗粒大小 $(20 \sim 50 \mathrm{~nm})$ 基本相同。 $\mathrm{TiO}_{2} / \mathrm{Ag}$ 的 $\mathrm{EDX}$ 数据(图 3(B)插图)中出 现了 $\mathrm{Ag}$ 元素, 说明 $\mathrm{Ag}$ 已经成功负载在 $\mathrm{TiO}_{2}$ 颗粒表 面。比较图 3(C F) 插图中的 $\mathrm{EDX}$ 数据发现 $\mathrm{TiO}_{2} / \mathrm{Ag}-\mathrm{Ag}_{2} \mathrm{O}(x)$ 中 $\mathrm{Ag}$ 元素的原子百分含量随炦烧
温度升高先逐渐降低, $\mathrm{TiO}_{2} / \mathrm{Ag}-\mathrm{Ag}_{2} \mathrm{O}(350)$ 达到最低, 然后又有所升高。这是由于在煅烧过程中 $\mathrm{TiO}_{2} / \mathrm{Ag}$ 样品中的 $\mathrm{Ag}$ 部分被氧化为 $\mathrm{Ag}_{2} \mathrm{O}$, 当温度达到 $350{ }^{\circ} \mathrm{C}$ 后, $\mathrm{Ag}_{2} \mathrm{O}$ 在高温环境中又迅速分解成 $\mathrm{Ag}$ 和 $\mathrm{O}_{2}$, 并且 $\mathrm{Ag}_{2} \mathrm{O}$ 分解的速度大于生成的速度 ${ }^{[22]}$ 。从 $\mathrm{TiO}_{2} / \mathrm{Ag}-\mathrm{Ag}_{2} \mathrm{O}(300$ )的 TEM 照片(图 3(G)) 可见, 在 $\mathrm{TiO}_{2}$ 颗粒表面均匀负载着尺寸约为 $5 \mathrm{~nm}$ 的 $\mathrm{Ag}$ 纳米 粒子(红色圆圈中的黑色颗粒)。其高分辨 TEM 照片 (图 3(H)) 中有 $\mathrm{TiO}_{2}(101)$ 面的晶格条纹, 间距为 $0.350 \mathrm{~nm}^{[12]}$, 以及 $\mathrm{Ag}(111)$ 面的晶格条纹, 间距为 $0.236 \mathrm{~nm}^{[22]}$ 。晶格在 $\mathrm{Ag}$ 纳米粒子边缘区域有微小 的变化, 主要源于在 $\mathrm{Ag}$ 表面原位氧化生成的 $\mathrm{Ag}_{2} \mathrm{O}$, 进一步证明成功制备了 $\mathrm{Ag}$ 和 $\mathrm{Ag}_{2} \mathrm{O}$ 共修饰的 $\mathrm{TiO}_{2}$ 复合光催化剂。
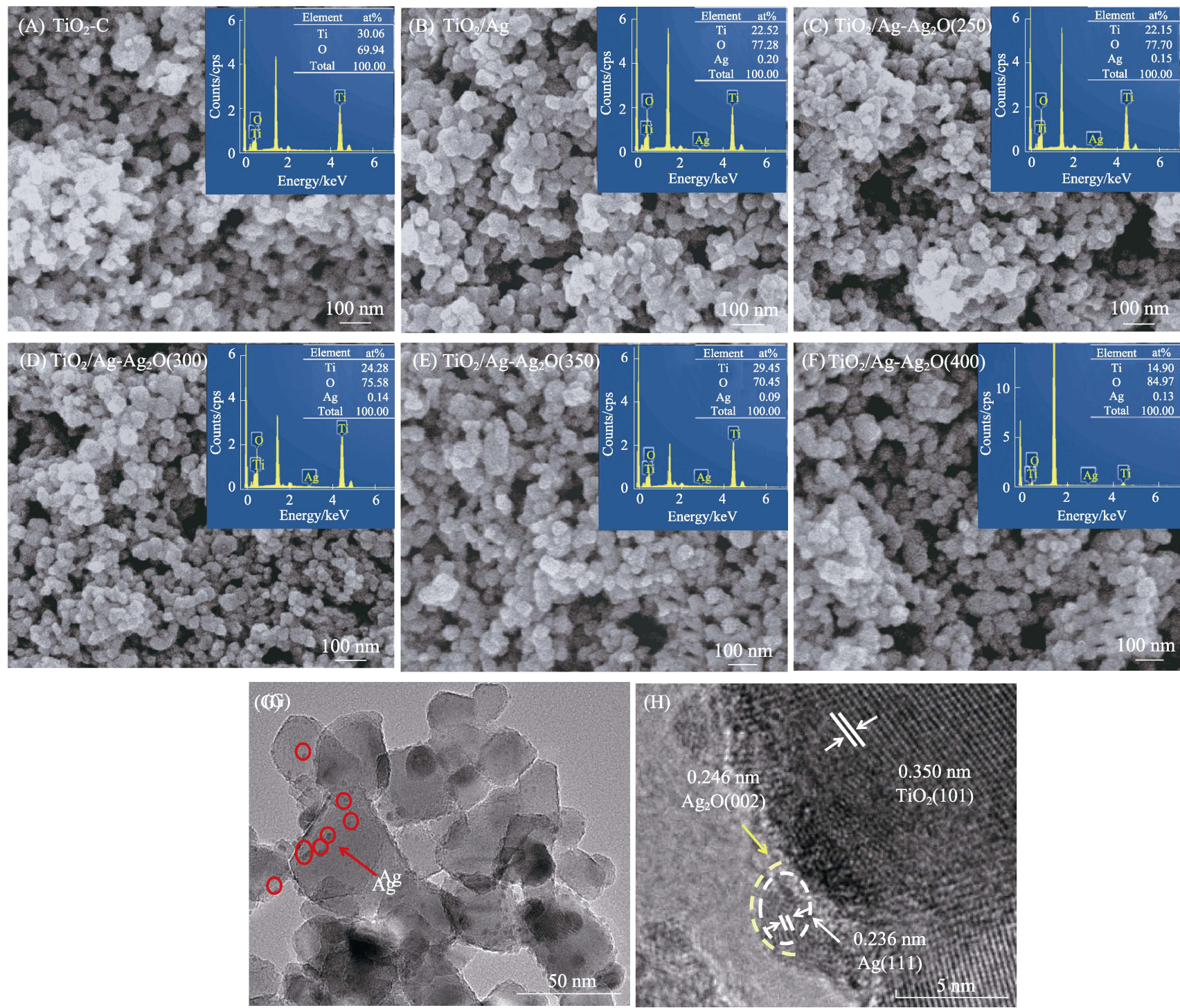

图 $3(\mathrm{~A} \sim \mathrm{F}) \mathrm{TiO}_{2}-\mathrm{C} 、 \mathrm{TiO}_{2} / \mathrm{Ag}$ 和 $\mathrm{TiO}_{2} / \mathrm{Ag}-\mathrm{Ag}_{2} \mathrm{O}(x)$ 的 FESEM 照片(插图: $\mathrm{EDX}$ 谱图和数据) 和 $(\mathrm{G}, \mathrm{H}) \mathrm{TiO}_{2} / \mathrm{Ag}-\mathrm{Ag}_{2} \mathrm{O}(300)$ 的 TEM 照片

Fig. 3 (A-F) FESEM images of $\mathrm{TiO}_{2}-\mathrm{C}, \mathrm{TiO}_{2} / \mathrm{Ag}$ and $\mathrm{TiO}_{2} / \mathrm{Ag}-\mathrm{Ag}_{2} \mathrm{O}(x)$ with insets showing their corresponding EDS spectra and data, and TEM images of $\mathrm{TiO}_{2} / \mathrm{Ag}-\mathrm{Ag}_{2} \mathrm{O}(300)$ at low $(\mathrm{G})$ and high $(\mathrm{H})$ magnifications 
图 4(A)是不同样品的 XPS 全谱图。位于 289、 473 和 $534 \mathrm{eV}$ 结合能的峰分别对应于 $\mathrm{C} 1 \mathrm{~s} 、 \mathrm{Ti2p}$ 和 $\mathrm{O} 1 \mathrm{~s}$ 的特征峰, 其中 $\mathrm{Ti} 、 \mathrm{O}$ 元素主要来源于 $\mathrm{TiO}_{2}, \mathrm{C}$ 元素则来源于碳污染。与 $\mathrm{TiO}_{2}-\mathrm{C}$ 相比, 在 $\mathrm{TiO}_{2} / \mathrm{Ag}$ 和 $\mathrm{TiO}_{2} / \mathrm{Ag}-\mathrm{Ag}_{2} \mathrm{O}(x)$ 中出现了 $\mathrm{Ag}$ 的特征峰, 并且在 $\mathrm{Ag} 3 \mathrm{~d}$ 高分辨率 XPS 图谱(图 4(B))中, $\mathrm{TiO}_{2} / \mathrm{Ag}$ 和 $\mathrm{TiO}_{2} / \mathrm{Ag}-\mathrm{Ag}_{2} \mathrm{O}$ 在大约 368 和 $374 \mathrm{eV}$ 处有明显的 $A g 3 d_{5 / 2}$ 和 $A g 3 d_{3 / 2}$ 特征峰, 说明存在 $A g$ 元素。 $\mathrm{TiO}_{2} / \mathrm{Ag}-\mathrm{Ag}_{2} \mathrm{O}(x)$ 与 $\mathrm{TiO}_{2} / \mathrm{Ag}$ 相比, 其特征峰向较高 的结合能偏移, 这是由于裧烧后部分 $\mathrm{Ag}$ 转变为 $\mathrm{Ag}_{2} \mathrm{O}$ 造成的 ${ }^{[23]}$ 。图 4(C) 是 $\mathrm{TiO}_{2} / \mathrm{Ag}-\mathrm{Ag}_{2} \mathrm{O}(300)$ 样品 的 Ag3d 典型峰拟合曲线。 367.44 和 $368.15 \mathrm{eV}$ 的峰 分别对应于 $\mathrm{Ag}^{0}$ 和 $\mathrm{Ag}^{+}$的 $3 \mathrm{~d}_{5 / 2}$ 轨道; 373.46 和 $374.16 \mathrm{eV}$ 的峰分别对应于 $\mathrm{Ag}^{0}$ 和 $\mathrm{Ag}^{+}$的 $3 \mathrm{~d}_{3 / 2}$ 轨道 ${ }^{[22,24]}$ 。图 4(D) 是 $\mathrm{TiO}_{2} / \mathrm{Ag}-\mathrm{Ag}_{2} \mathrm{O}(300)$ 样品的 $\mathrm{O} 1 \mathrm{~s}$ 分峰的拟合曲线, 其中 $\mathrm{Ti}-\mathrm{O}$ 和 $\mathrm{Ti}-\mathrm{O}-\mathrm{Ag}$ 键的峰对应于 $\mathrm{Ag}$ 与 $\mathrm{TiO}_{2}$ 之 间的作用力。而且, 在 XPS 图谱的 Ag 分峰数据(表 1)中, $\mathrm{TiO}_{2} / \mathrm{Ag}-\mathrm{Ag}_{2} \mathrm{O}(200 \sim 400)$ 的 $\mathrm{Ag}^{+} / \mathrm{Ag}^{0}$ 数值先升 后降, 进一步证明 $\mathrm{TiO}_{2} / \mathrm{Ag}-\mathrm{Ag}_{2} \mathrm{O}$ 样品中同时存在 $\mathrm{Ag}$ 和 $\mathrm{Ag}_{2} \mathrm{O}$ 。

图 5 为不同样品紫外-可见漫反射光谱转化后 的吸收光谱图, 插图为对应样品的照片。 $\mathrm{TiO}_{2}-\mathrm{C}$ 光 催化剂样品的紫外-可见吸收光谱的吸收边在 $410 \mathrm{~nm}$
左右, 样品呈白色 ${ }^{[12]}$ 。 $\mathrm{TiO}_{2} / \mathrm{Ag}$ 光催化剂在 $410 \sim 800 \mathrm{~nm}$ 范围的可见光区具有较强的吸收, 白色的 $\mathrm{TiO}_{2}$ 样品 转变为棕红色。这主要是因为 $\mathrm{Ag}$ 纳米粒子的表面 等离子体共振效应引起了可见光区的吸收加强 ${ }^{[21]}$ 。 $\mathrm{TiO}_{2} / \mathrm{Ag}-\mathrm{Ag}_{2} \mathrm{O}$ 样品在紫外光区的吸收与 $\mathrm{TiO}_{2}-\mathrm{C}$ 无 明显差异, 在可见光区的吸收随着煅烧温度的升高 出现明显减弱, 分别呈紫色 $\left(\mathrm{TiO}_{2} / \mathrm{Ag}-\mathrm{Ag}_{2} \mathrm{O}(200)\right)$ 、暗蓝 色 $\left(\mathrm{TiO}_{2} / \mathrm{Ag}-\mathrm{Ag}_{2} \mathrm{O}(300)\right)$ 和灰白色 $\left(\mathrm{TiO}_{2} / \mathrm{Ag}-\mathrm{Ag}_{2} \mathrm{O}(400)\right)$ 。 随着烊烧温度的升高, $\mathrm{TiO}_{2} / \mathrm{Ag}-\mathrm{Ag}_{2} \mathrm{O}$ 样品表面的 $\mathrm{Ag}$ 逐渐被氧化为 $\mathrm{Ag}_{2} \mathrm{O}$, 导致 $\mathrm{Ag}$ 的等离子体共振效应 随之减弱。相比于 $\mathrm{TiO}_{2}, \mathrm{TiO}_{2} / \mathrm{Ag}$ 和 $\mathrm{TiO}_{2} / \mathrm{Ag}-\mathrm{Ag}_{2} \mathrm{O}(x)$ 样品的紫外吸收边略有红移, 可能是由于金属 $\mathrm{Ag}$ 与 $\mathrm{TiO}_{2}$ 之间形成了 $\mathrm{Ti}-\mathrm{O}-\mathrm{Ag}$ 键, 以及金属 $\mathrm{Ag}$ 被氧 化成 $\mathrm{Ag}_{2} \mathrm{O}$, 形成 $\mathrm{Ag}-\mathrm{O}-\mathrm{Ag}$ 键, 导致光催化剂体系跃 迁能量略有下降。而且, 相比于 $\mathrm{TiO}_{2}, \mathrm{TiO}_{2} / \mathrm{Ag}-\mathrm{Ag}_{2} \mathrm{O}$ 具有更强的紫外-可见光吸收性能。

\section{3 光催化制氢性能和光催化制氢机理}

图 6(A) 是不同样品的制氢速率柱状图。 $\mathrm{TiO}_{2} / \mathrm{Ag}-\mathrm{Ag}_{2} \mathrm{O}$ 的制氢速率随着样品煅烧温度的逐 步升高而表现出先升高后下降的规律, 并在煅烧温 度为 $300{ }^{\circ} \mathrm{C}$ 时达到最大 $(75.20 \mu \mathrm{mol} / \mathrm{h})$, 分别是 $\mathrm{TiO}_{2}-\mathrm{C}(3.59 \mu \mathrm{mol} / \mathrm{h})$ 和 $\mathrm{TiO}_{2} / \mathrm{Ag}(41.13 \mu \mathrm{mol} / \mathrm{h})$ 的制氢 速率的 21.0 和 1.8 倍。当煅烧温度超过 $300{ }^{\circ} \mathrm{C}$ 后样
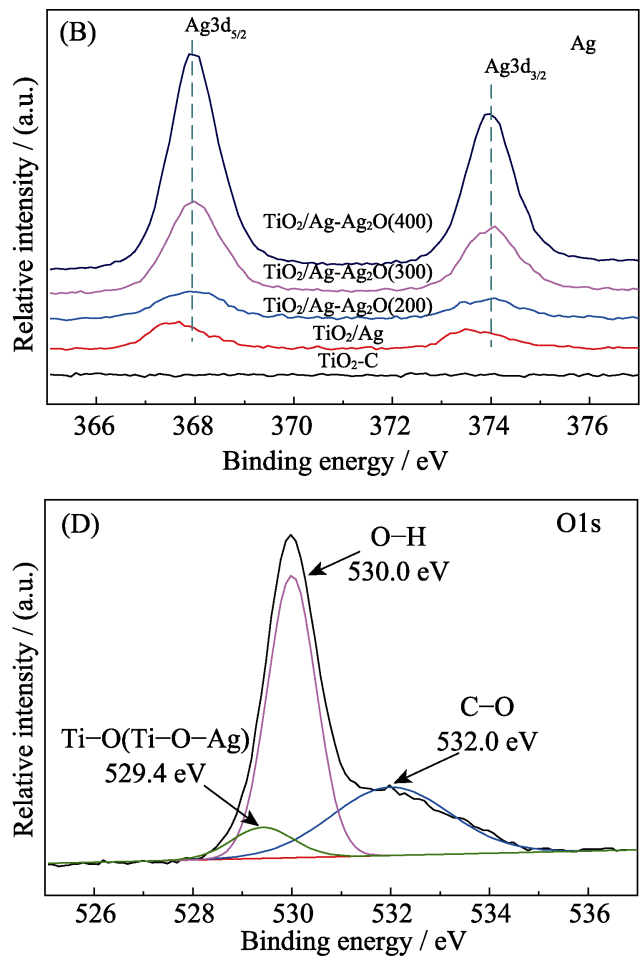

图 $4 \mathrm{TiO}_{2}-\mathrm{C} 、 \mathrm{TiO}_{2} / \mathrm{Ag}$ 和 $\mathrm{TiO}_{2} / \mathrm{Ag}-\mathrm{Ag}_{2} \mathrm{O}(x)$ 的(A)XPS 全谱和(B)Ag3d 谱的高分辨率 XPS 谱; $\mathrm{TiO}_{2} / \mathrm{Ag}-\mathrm{Ag}_{2} \mathrm{O}(300)$ 样品的(C)Ag3d 和(D)O1s 典型峰的拟合曲线

Fig. 4 (A) XPS survey spectra and (B) the high-resolution XPS spectra of $\mathrm{Ag} 3 \mathrm{~d}$ spectra of $\mathrm{TiO}_{2}-\mathrm{C}, \mathrm{TiO}_{2} / \mathrm{Ag}$ and $\mathrm{TiO}_{2} / \mathrm{Ag}-\mathrm{Ag}_{2} \mathrm{O}(x)$, and typical fitting curves of (C)Ag3d and (D)O1s for $\mathrm{TiO}_{2} / \mathrm{Ag}-\mathrm{Ag}_{2} \mathrm{O}(300)$ 
表 1 不同样品 XPS 的元素含量

Table 1 Contents of elements in various samples according to XPS analysis

\begin{tabular}{cccccc}
\hline Element & $\mathrm{TiO}_{2}-\mathrm{C}$ & $\mathrm{TiO}_{2} / \mathrm{Ag}$ & $\mathrm{TiO}_{2} / \mathrm{Ag}-\mathrm{Ag}_{2} \mathrm{O}(200)$ & $\mathrm{TiO}_{2} / \mathrm{Ag}-\mathrm{Ag}_{2} \mathrm{O}(300)$ & $\mathrm{TiO} / \mathrm{Ag}-\mathrm{Ag}{ }_{2} \mathrm{O}(400)$ \\
\hline $\mathrm{C} 1 \mathrm{~s} / \%$ & 38.97 & 25.82 & 46.37 & 37.05 & 28.11 \\
$\mathrm{Ti} 2 \mathrm{p} / \%$ & 19.53 & 25.01 & 15.18 & 19.39 & 22.73 \\
$\mathrm{O} 1 \mathrm{~s} / \%$ & 41.50 & 48.82 & 38.05 & 42.43 & 46.46 \\
$\mathrm{Ag} 3 \mathrm{~d} / \%$ & - & 0.36 & 0.40 & 1.13 & 2.70 \\
$\mathrm{Ag}^{+} / \mathrm{Ag}^{0}$ & - & 1.02 & 2.15 & 4.42 & 3.52 \\
\hline
\end{tabular}

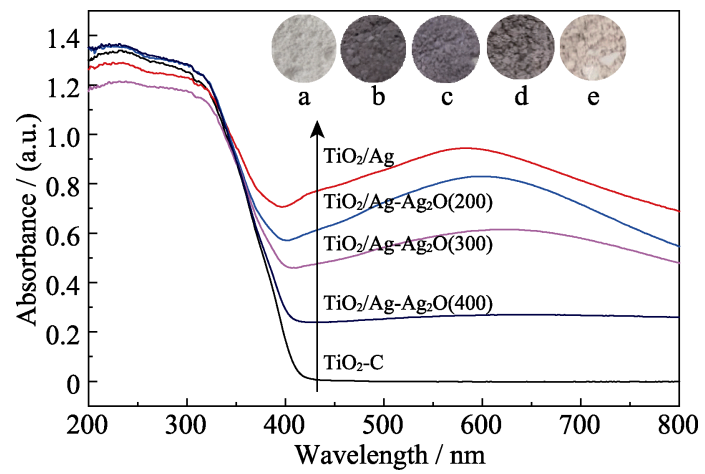

图 $5 \mathrm{TiO}_{2}-\mathrm{C} 、 \mathrm{TiO}_{2} / \mathrm{Ag}$ 和 $\mathrm{TiO}_{2} / \mathrm{Ag}-\mathrm{Ag}_{2} \mathrm{O}(x)$ 的 UV-Vis 吸收 光谱及对应样品照片

Fig. 5 UV-Vis absorption spectra of $\mathrm{TiO}_{2}-\mathrm{C}, \mathrm{TiO}_{2} / \mathrm{Ag}$ and $\mathrm{TiO}_{2} / \mathrm{Ag}-\mathrm{Ag}_{2} \mathrm{O}(x)$ and their corresponding photographs (inset) (a) $\mathrm{TiO}_{2}-\mathrm{C}$; (b) $\mathrm{TiO}_{2} / \mathrm{Ag}$; (c) $\mathrm{TiO}_{2} / \mathrm{Ag}-\mathrm{Ag}_{2} \mathrm{O}(200)$; (d) $\mathrm{TiO}_{2} / \mathrm{Ag}-$ $\mathrm{Ag}_{2} \mathrm{O}(300)$; (e) $\mathrm{TiO}_{2} / \mathrm{Ag}-\mathrm{Ag}_{2} \mathrm{O}(400)$
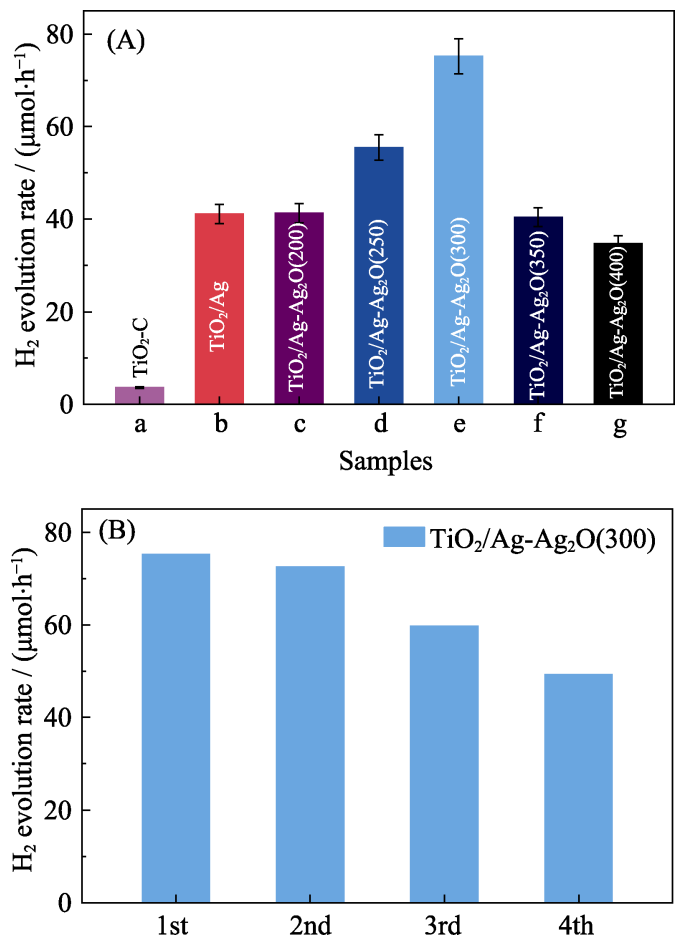

图 6 (A) $\mathrm{TiO}_{2}-\mathrm{C} 、 \mathrm{TiO}_{2} / \mathrm{Ag}$ 和 $\mathrm{TiO}_{2} / \mathrm{Ag}-\mathrm{Ag}_{2} \mathrm{O}(x)$ 的光催化制 氢性能; (B) $\mathrm{TiO}_{2} / \mathrm{Ag}-\mathrm{Ag}_{2} \mathrm{O}(300)$ 的循环性能

Fig. 6 (A) Photocatalytic $\mathrm{H}_{2}$-evolution activity of $\mathrm{TiO}_{2}-\mathrm{C}$, $\mathrm{TiO}_{2} / \mathrm{Ag}$ and $\mathrm{TiO}_{2} / \mathrm{Ag}-\mathrm{Ag}_{2} \mathrm{O}(x)$, and (B) cycling performance of $\mathrm{TiO}_{2} / \mathrm{Ag}-\mathrm{Ag}_{2} \mathrm{O}(300)$
品的制氢速率有所下降。不同殷烧温度的样品制氢 性能变化规律和 EDX 图谱(图 3(A F) 插图)中 $\mathrm{Ag}$ 和 $\mathrm{Ag}_{2} \mathrm{O}$ 的含量变化趋势相对应, 说明表面负载 $\mathrm{Ag}$ 和 $\mathrm{Ag}_{2} \mathrm{O}$ 对 $\mathrm{TiO}_{2}$ 光催化剂制氢性能具有重要影响。为 了验证光催化剂的性能稳定性, 测试了样品 $\mathrm{TiO}_{2} / \mathrm{Ag}-\mathrm{Ag}_{2} \mathrm{O}(300)$ 的 4 次循环性能(图 6(B))。由图 可知, 随着循环次数的增加, 样品 $\mathrm{TiO}_{2} / \mathrm{Ag}-\mathrm{Ag}_{2} \mathrm{O}(300)$ 的制氢速率虽有所下降, 但始终高于 $\mathrm{TiO}_{2} / \mathrm{Ag}$ 和 $\mathrm{TiO}_{2}-\mathrm{C}$, 说明 $\mathrm{TiO}_{2} / \mathrm{Ag}-\mathrm{Ag}_{2} \mathrm{O}$ 样品具有一定的稳定性。

图 7 为 $\mathrm{TiO}_{2} / \mathrm{Ag}-\mathrm{Ag}_{2} \mathrm{O}$ 的光催化制氢机理示意 图。在紫外光照射下, $\mathrm{TiO}_{2}$ 产生光生电子和空穴, 金 属 $\mathrm{Ag}$ 充当电子助剂能够快速转移光生电子, 加快 光生电子-空穴对的有效分离, 抑制两者的复合。Ag 表面的光生电子进一步转移到其上的 $\mathrm{Ag}_{2} \mathrm{O}$, 同时 $\mathrm{Ag}_{2} \mathrm{O}$ 作为界面催化反应活性位点吸附溶液中的 $\mathrm{H}^{+}$

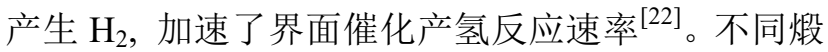
烧温度样品制氢性能变化规律与样品 $\mathrm{Ag}_{2} \mathrm{O}$ 含量变 化规律的一致性也证明了这一点。所以, 电子助剂 $\mathrm{Ag}$ 和界面活性位点 $\mathrm{Ag}_{2} \mathrm{O}$ 的协同作用增强了 $\mathrm{TiO}_{2}$ 光催化的制氢性能。

为验证 $\mathrm{TiO}_{2} / \mathrm{Ag}-\mathrm{Ag}_{2} \mathrm{O}$ 光催化制氢机理, 对样品 进行光电化学表征。图 8(A)是不同样品的线性扫描 伏安曲线( $\mathrm{LSV})$ 。其中, $\mathrm{TiO}_{2} / \mathrm{Ag}$ 的电流密度明显超 过 $\mathrm{TiO}_{2}-\mathrm{C}$, 产氢过电位略微降低, 表明 $\mathrm{Ag}$ 作为助 催化剂能够快速捕获光生电子, 促使溶液中的 $\mathrm{H}^{+}$ 还原成 $\mathrm{H}_{2}{ }^{[21]}$ 。 $\mathrm{TiO}_{2} / \mathrm{Ag}-\mathrm{Ag}_{2} \mathrm{O}$ 的电流密度进一步增 大, 同时产氢过电压逐渐降低, 表明 $\mathrm{Ag}_{2} \mathrm{O}$ 是一种 高效的界面催化活性位点, 能有效吸附溶液中的 $\mathrm{H}^{+}$, 加快界面催化反应。为了进一步研究 $\mathrm{TiO}_{2} / \mathrm{Ag}-\mathrm{Ag}_{2} \mathrm{O}$ 中光生载流子的捕获、分离和传输效率, 测量了不 同光催化剂的瞬态光电流响应曲线(图 8(B))和电 化学阻抗谱(EIS, 图 8(C))。由图 8(B) 可以看出, 相对于 $\mathrm{TiO}_{2}-\mathrm{C}, \mathrm{TiO}_{2} / \mathrm{Ag}$ 的光电流密度明显提升, 说明 $\mathrm{Ag}$ 具有优异的载流子传输性能, 能有效促进 光生电荷的分离。随着㷽烧温度从 $200{ }^{\circ} \mathrm{C}$ 升到 $400{ }^{\circ} \mathrm{C}, \mathrm{TiO}_{2} / \mathrm{Ag}-\mathrm{Ag}_{2} \mathrm{O}(x)$ 的光电流密度先明显增 加, $\mathrm{TiO}_{2} / \mathrm{Ag}-\mathrm{Ag}_{2} \mathrm{O}(300)$ 达到最大, 后有所下降, 但 


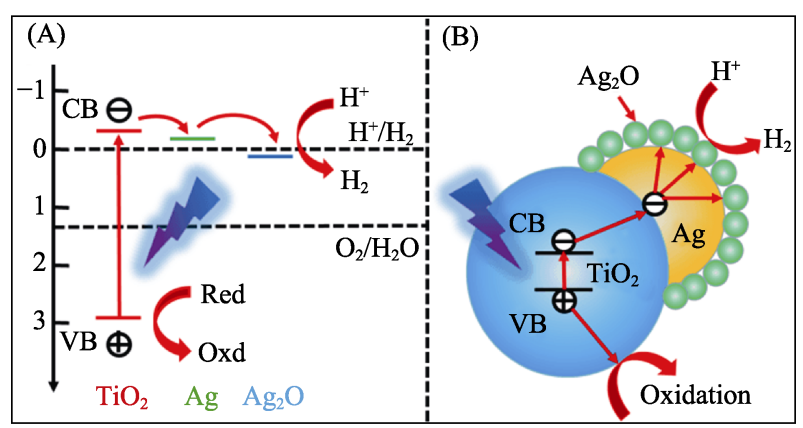

图 $7 \mathrm{TiO}_{2} / \mathrm{Ag}-\mathrm{Ag}_{2} \mathrm{O}$ 的光催化制氢机理示意图

Fig. 7 Schematic diagram of photocatalytic $\mathrm{H}_{2}$-evolution mechanism of $\mathrm{TiO}_{2} / \mathrm{Ag}-\mathrm{Ag}_{2} \mathrm{O}$
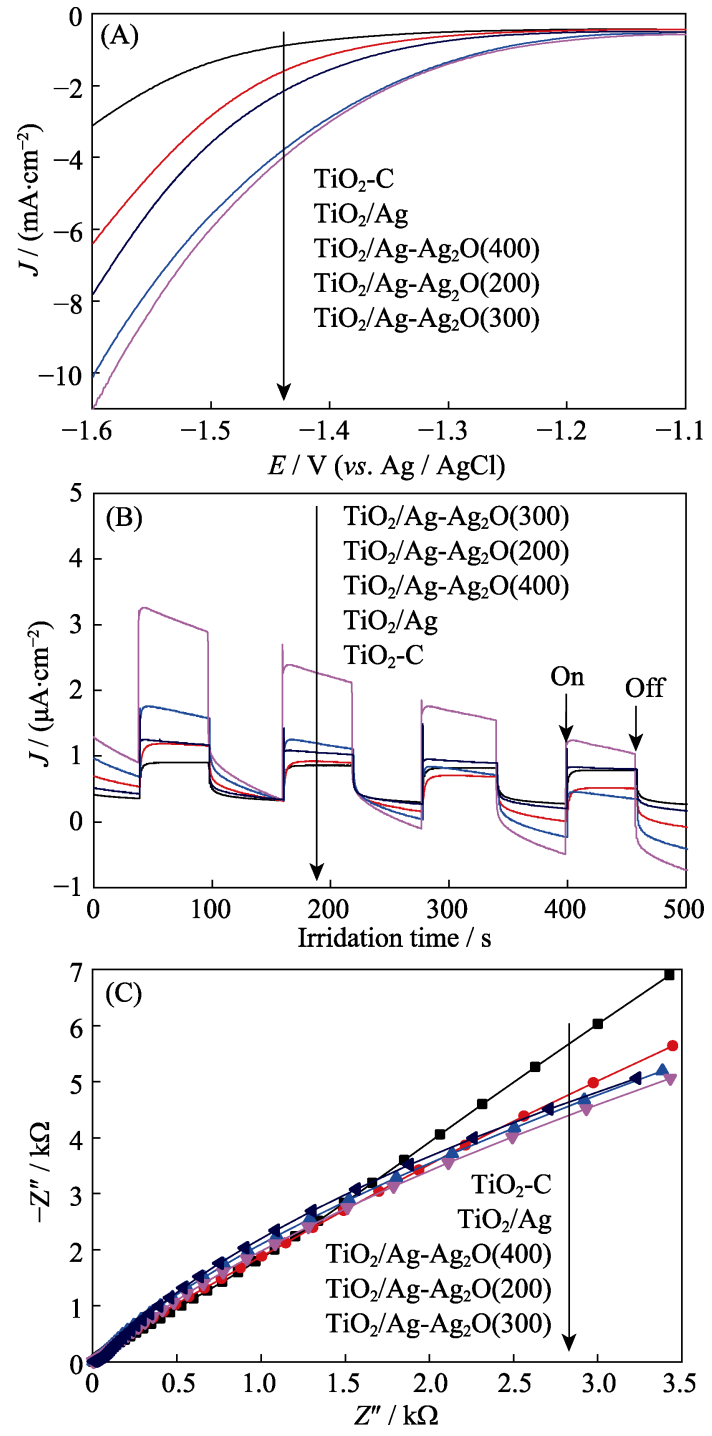

图 $8 \mathrm{TiO}_{2}-\mathrm{C} 、 \mathrm{TiO}_{2} / \mathrm{Ag}$ 和 $\mathrm{TiO}_{2} / \mathrm{Ag}-\mathrm{Ag}_{2} \mathrm{O}(x)$ 的(A)线性扫描曲 线(LSV)、(B)瞬态光电流响应和(C)电化学阻抗谱(EIS)

Fig. 8 (A) Linear sweep voltammetry (LSV) curves, (B) transient photocurrent responses, and $(\mathrm{C})$ electrochemical impedance spectra (EIS) of $\mathrm{TiO}_{2}-\mathrm{C}, \mathrm{TiO}_{2} / \mathrm{Ag}$ and $\mathrm{TiO}_{2} / \mathrm{Ag}-\mathrm{Ag}_{2} \mathrm{O}(x)$

$\mathrm{TiO}_{2} / \mathrm{Ag}-\mathrm{Ag}_{2} \mathrm{O}(400)$ 的光电流密度依然超过 $\mathrm{TiO}_{2}-\mathrm{C}$ 。 由图 8(C) 可见, $\mathrm{TiO}_{2} / \mathrm{Ag}-\mathrm{Ag}_{2} \mathrm{O}(x)$ 样品的圆弧半径小 于 $\mathrm{TiO}_{2}-\mathrm{C}$ 和 $\mathrm{TiO}_{2} / \mathrm{Ag}$, 说明 $\mathrm{Ag}_{2} \mathrm{O}$ 与 $\mathrm{Ag}$ 之间的传输

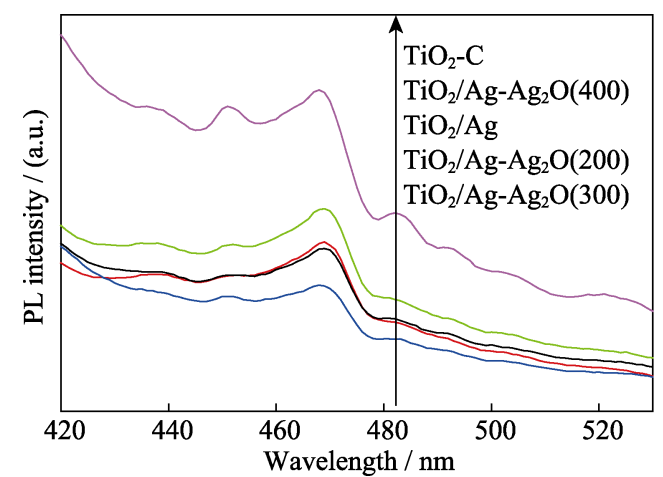

图 $9 \mathrm{TiO}_{2}-\mathrm{C} 、 \mathrm{TiO}_{2} / \mathrm{Ag}$ 和 $\mathrm{TiO}_{2} / \mathrm{Ag}-\mathrm{Ag}_{2} \mathrm{O}(x)$ 的苂光光谱图 Fig. 9 Photoluminescence (PL) spectra of $\mathrm{TiO}_{2}-\mathrm{C}, \mathrm{TiO}_{2} / \mathrm{Ag}$ and $\mathrm{TiO}_{2} / \mathrm{Ag}-\mathrm{Ag}_{2} \mathrm{O}(x)$

阻力小, 光生载流子的传输效率高 ${ }^{[25]}$ 。 $\mathrm{Ag}$ 与 $\mathrm{Ag}_{2} \mathrm{O}$ 以适当比例共存时, 电子助剂 $\mathrm{Ag}$ 和界面活性位点 $\mathrm{Ag}_{2} \mathrm{O}$ 能显著提高光生电子转移速度, 加快界面的 产氢催化反应速率, 促进光生电子和空穴的分离, 改善 $\mathrm{TiO}_{2}$ 光催化制氢性能。

为揭示样品在光照时光生载流子复合的情况, 测定了样品的苂光(PL)光谱图(图 9)。相比于 $\mathrm{TiO}_{2}-\mathrm{C}$, $\mathrm{TiO}_{2} / \mathrm{Ag}$ 的苂光强度有所下降, 说明电子和空穴的 复合得到有效抑制, 主要是由于 $\mathrm{Ag}$ 起到了快速传 输电子的作用。 $\mathrm{TiO}_{2} / \mathrm{Ag}-\mathrm{Ag}_{2} \mathrm{O}(x)$ 的光生载流子复合 的情况相对于 $\mathrm{TiO}_{2} / \mathrm{Ag}$ 有增有减。 $\mathrm{TiO}_{2} / \mathrm{Ag}-\mathrm{Ag}_{2} \mathrm{O}(300)$ 的荧光强度最弱, 说明其电子空穴复合率最低、分 离效率最高 ${ }^{[26]}$, 有助于改善产氢性能。

\section{3 结论}

本研究采用光沉积法和㷽烧法制备了 $\mathrm{TiO}_{2} / \mathrm{Ag}-\mathrm{Ag}_{2} \mathrm{O}$ 复合光催化剂, 表现出较高的光催化 制氢性能。 $300{ }^{\circ} \mathrm{C}$ 炦烧制得的复合光催化剂的制氢 速率达到最大值 $(75.20 \mu \mathrm{mol} / \mathrm{h})$, 分别是 $\mathrm{TiO}_{2}-\mathrm{C}$ 和 $\mathrm{TiO}_{2} / \mathrm{Ag}$ 的 21.0 和 1.8 倍。 $\mathrm{TiO}_{2} / \mathrm{Ag}-\mathrm{Ag}_{2} \mathrm{O}$ 复合光催 化剂制氢性能显著提高的原因是: $\mathrm{Ag}$ 作为电子助剂 快速转移光生电子, $\mathrm{Ag}_{2} \mathrm{O}$ 作为界面催化反应活性位 点加快界面产氢速率，两者的协同作用促使光生电 子-空穴复合率显著降低，从而提高了 $\mathrm{TiO}_{2}$ 的制氢 速率。本研究对设计具有高效制氢性能的 $\mathrm{TiO}_{2}$ 光催 化复合材料具有一定的指导意义。

\section{参考文献:}

[1] DU H, LIU Y, SHENG C, et al. Nanoheterostructured photocatalysts for improving photocatalytic hydrogen production. Chinese Journal of Catalysis, 2017, 38(8): 1295-1306.

[2] ZHANG K, PARK J H. Surface localization of defects in black $\mathrm{TiO}_{2}$ : enhancing photoactivity or reactivity. Journal of Physical 
Chemistry C, 2017, 8: 199-207.

[3] LIU Q, SHEN J, YU X, et al. Unveiling the origin of boosted photocatalytic hydrogen evolution in simultaneously $(\mathrm{S}, \mathrm{P}, \mathrm{O})$ codoped and exfoliated ultrathin $\mathrm{g}_{-} \mathrm{C}_{3} \mathrm{~N}_{4}$ nanosheets. Applied $\mathrm{Ca}$ talysis B: Environmental, 2019, 248: 84-94.

[4] TIAN L, YANG X, LIU Q, et al. Anchoring metal-organic framework nanoparticles on graphitic carbon nitrides for solar-driven photocatalytic hydrogen evolution. Applied Surface Science, 2018, 455: 403-409.

[5] MA Y, LI Q. Preparation and characterization of $\mathrm{TiO}_{2} / \mathrm{Co}_{3} \mathrm{O}_{4}$ nanocomposites and their photocatalytic activity for hydrogen evolution. Journal of Inorganic Materials, 2016, 31(8): 841-844.

[6] JIANG Y, QUA F, TIAN L, et al. Self-assembled g- $\mathrm{C}_{3} \mathrm{~N}_{4}$ nanoarchitectures with boosted photocatalytic solar-to-hydrogen efficiency. Applied Surface Science, 2019, 487: 59-67.

[7] WEI J, LI X, WANG H, et al. Nitrogen doped carbon quantum dots/titanium dioxide composites for hydrogen evolution under sunlight. Journal of Inorganic Materials, 2015, 30(9): 925-930.

[8] YAN C, XUE X, ZHANG W, et al. Well-designed Te/SnS $2 / \mathrm{Ag}$ artificial nanoleaves for enabling and enhancing visible-light driven overall splitting of pure water. Nano Energy, 2017, 39: 539-545.

[9] LIU W, SHEN J, LIU Q, et al. Porous MoP network structure as co-catalyst for $\mathrm{H}_{2}$ evolution over $\mathrm{g}-\mathrm{C}_{3} \mathrm{~N}_{4}$ nanosheets. Applied Surface Science, 2018, 462: 822-830.

[10] TANG H, WANG R, ZHAO C, et al. Oxamide-modified g- $\mathrm{C}_{3} \mathrm{~N}_{4}$ nanostructures: tailoring surface topography for high-performance visible light photocatalysis. Chemical Engineering Journal, 2019, 374: 1064-1075.

[11] LI C, JIN H, YANG Z, et al. Preparation and photocatalytic properties of mesoporous $\mathrm{RGO} / \mathrm{TiO}_{2}$ composites. Journal of Inorganic Materials, 2017, 32 (04): 357-364.

[12] WANG P, LU Y, WANG X, et al. Co-modification of amorphous$\mathrm{Ti}(\mathrm{IV})$ hole cocatalyst and $\mathrm{Ni}(\mathrm{OH})_{2}$ electron cocatalyst for enhanced photocatalytic $\mathrm{H}_{2}$-production performance of $\mathrm{TiO}_{2}$. Applied Surface Science, 2017, 391: 259-266.

[13] ZHANG W, ZHANG H, XU J, et al. 3D flower-like heterostructured $\mathrm{TiO}_{2} @ \mathrm{Ni}(\mathrm{OH})_{2}$ microspheres for solar photocatalytic hydrogen production. Chinese Journal of Catalysis, 2019, 40(3): 320-325.

[14] KUMARAVEL V, MATHEW S, BARTIETT J, et al. Photocatalytic hydrogen production using metal doped $\mathrm{TiO}_{2}$ : a review of recent advances. Applied Catalysis B: Environmental, 2019, 244: 1021-1064.

[15] ZHAO D, YANG C F. Recent advances in the $\mathrm{TiO}_{2} / \mathrm{CdS}$ nano- composite used for photocatalytic hydrogen production and quantum-dot-sensitized solar cells. Renewable and Sustainable Energy Reviews, 2016, 54: 1048-1059.

[16] CHEN F, LUO W, Mo Y, et al. In situ photodeposition of amorphous $\mathrm{CoS}_{x}$ on the $\mathrm{TiO}_{2}$ towards hydrogen evolution. Applied Surface Science, 2018, 430: 448-456.

[17] GUPTA B, MELVIN A A, MATTHEWS T, et al. $\mathrm{TiO}_{2}$ modification by gold $(\mathrm{Au})$ for photocatalytic hydrogen $\left(\mathrm{H}_{2}\right)$ production. Renewable and Sustainable Energy Reviews, 2016, 58: 1366-1375.

[18] HOU L, ZHANG M, GUAN Z, et al. Effect of platinum dispersion on photocatalytic performance of $\mathrm{Pt}_{-} \mathrm{TiO}_{2}$. Journal of Nanoparticle Research, 2018, 20(3): 1-8.

[19] SARAVANAN R, MANOJ D, QIN J, et al. Mechanothermal synthesis of $\mathrm{Ag} / \mathrm{TiO}_{2}$ for photocatalytic methyl orange degradation and hydrogen production. Process Safety and Environmental Protection, 2018, 120: 339-347.

[20] WANG P, SHENG Y, WANG F, et al. Synergistic effect of electrontransfer mediator and interfacial catalytic active-site for the enhanced $\mathrm{H}_{2}$ evolution performance: a case study of $\mathrm{CdS} / \mathrm{Au}$ photocatalyst. Applied Catalysis B: Environmentai, 2018, 220: 561-569.

[21] YU H, LIU W, WANG X, et al. Promoting the interfacial $\mathrm{H}_{2}$-evolution reaction of metallic $\mathrm{Ag}$ by $\mathrm{Ag}_{2} \mathrm{~S}$ cocatalyst: a case study of $\mathrm{TiO}_{2} / \mathrm{Ag}-\mathrm{Ag}_{2} \mathrm{~S}$ photocatalyst. Applied Catalysis B: Environmental, 2018, 225: 415-423.

[22] WANG X, LIAO D, YU H, et al. Highly efficient $\mathrm{BiVO}_{4}$ singlecrystal photocatalyst with selective $\mathrm{Ag}_{2} \mathrm{O}-\mathrm{Ag}$ modification: orientation transport, rapid interfacial transfer and catalytic reaction. Dalton Transactions, 2018, 47(18): 6370-6377.

[23] YU H, LIU R, WANG X, et al. Enhanced visible-light photocatalytic activity of $\mathrm{Bi}_{2} \mathrm{WO}_{6}$ nanoparticles by $\mathrm{Ag}_{2} \mathrm{O}$ cocatalyst. Applied Catalysis B: Environmental, 2012, 111-112: 326-333.

[24] LI J, HAO H, ZHOU J, et al. $\mathrm{Ag} @ \mathrm{AgCl}$ QDs decorated g- $\mathrm{C}_{3} \mathrm{~N}_{4}$ nanoplates: the photoinduced charge transfer behavior under visible light and full arc irradiation. Applied Surface Science, 2017, 422: 626-637.

[25] KIM J, JUN H, HONG S, et al. Charge transfer in iron photoanode modified with carbon nanotubes for photoelectrochemical water oxidation: an electrochemical impendence study. International Journal of Hydrogen Energy, 2011, 36: 9462-9468.

[26] LIU Y, DING S, SHI Y, et al. Construction of $\mathrm{CdS} / \mathrm{CoO}_{x}$ core-shell nanorods for efficient photocatalytic $\mathrm{H}_{2}$ evolution. Applied Catalysis B: Environmental, 2018, 234: 106-116. 